\title{
First-Principles Study of Carbon Vacancy in $\mathrm{Ta}_{4} \mathrm{AlC}_{3}$
}

\author{
Y. L. Du ${ }^{1,2}$, Z. M. Sun ${ }^{1, *}$, H. Hashimoto ${ }^{1}$ and W. B. Tian ${ }^{1}$ \\ ${ }^{1}$ Materials Research Institute for Sustainable Development, National Institute of Advanced Industrial Science \\ and Technology (AIST), Nagoya 463-8560, Japan \\ ${ }^{2}$ Department of Materials Science and Engineering, Nanjing University of Science and Technology, \\ Nanjing 210094, P.R.China
}

We have investigated the carbon mono-vacancy in $\mathrm{Ta}_{4} \mathrm{AlC}_{3}$ by first-principles calculations. We identify the $2 a$ sites as the probable carbon vacancy sites in $\mathrm{Ta}_{4} \mathrm{AlC}_{3}$ based on the energetics of vacancy formation. It was found that introducing carbon vacancies decreased the phase stability of $\mathrm{Ta}_{4} \mathrm{AlC}_{3}$. There are no significant changes in volume by introducing carbon vacancy. However, the bulk modulus decreased when introducing carbon vacancy. The differences in density of states of $\mathrm{Ta}_{4} \mathrm{AlC}_{3}$ with and without carbon vacancies are analyzed. Some vacancy peaks near the Fermi level were observed. Additionally, the density of states at the Fermi level increases by introducing carbon vacancies, which may be of benefit to the electron transport in $\mathrm{Ta}_{4} \mathrm{AlC}_{3}$. [doi:10.2320/matertrans.MAW200803]

(Received April 7, 2008; Accepted May 26, 2008; Published July 9, 2008)

Keywords: carbon mono-vacancy, $\mathrm{Ta}_{4} \mathrm{AlC} \mathrm{C}_{3}$, formation energy, first-principles

\section{Introduction}

MAX phases $\left(\mathrm{M}_{n+1} \mathrm{AX}_{n}\right.$, where $n=1,2$ or $3, \mathrm{M}$ is an early transition metal, $\mathrm{A}$ is an A-group element, and $\mathrm{X}$ is either $\mathrm{C}$ or $\mathrm{N}$ ) are a family of layered ternary compounds, which have attracted considerable attention due to their unique combination of metallic and ceramic properties. ${ }^{1-3)}$ According to the value of $n$, MAX phases are classified into three groups, i.e. 211, 312 and 413 phases. For a long time, $\mathrm{Ti}_{4} \mathrm{AlN}_{3}$ is believed to be the only member of 413 phases. ${ }^{4)}$ As a result, research on 413 phases is not as intensive as research on 211 and 312 phases. However, recently, important progresses on 413 phases were reported in Ta-Al-C system, in which $\mathrm{Ta}_{4} \mathrm{AlC}_{3}$ bulk materials were synthesized either in the form of polycrystals ${ }^{5-9)}$ or single-crystals. ${ }^{10)}$ The structural, mechanical and physical properties of $\mathrm{Ta}_{4} \mathrm{AlC}_{3}$ have been studied. ${ }^{5-11)}$ It was found that $\mathrm{Ta}_{4} \mathrm{AlC}_{3}$ exhibits a combination of improved mechanical properties, good damage tolerance and excellent thermal shock resistance, which makes it a promising technological material.

It is known that many MAX phases usually exist in substoichiometric form with a substantial amount of carbon or nitrogen vacancies. For example, the nitrogen vacancies in $\mathrm{Ti}_{4} \mathrm{AlN}_{3}$ have been found and studied experimentally and theoretically. ${ }^{12,13)}$ These nitrogen vacancies have remarkable influence on structural, mechanical and physical properties. At present, the defect formation in $\mathrm{Ta}_{4} \mathrm{AlC}_{3}$ has not been investigated. In this paper, we studied the carbon monovacancy in $\mathrm{Ta}_{4} \mathrm{AlC}_{3}$ by first-principles calculations.

\section{Details of Calculation}

We have used density functional theory implemented into the WIEN2K code for the calculations in the present work. ${ }^{14)}$ The generalized gradient approximation (GGA) exchange correlation functional of Perdew, Burke, and Ernzerhof was used. Detailed computational procedures can be found in our previous works, in which we studied the electronic structure

*Corresponding author, E-mail: z.m.sun@aist.go.jp

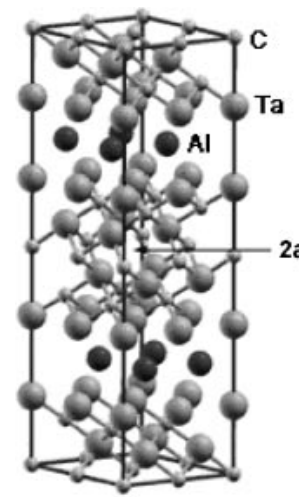

(a)

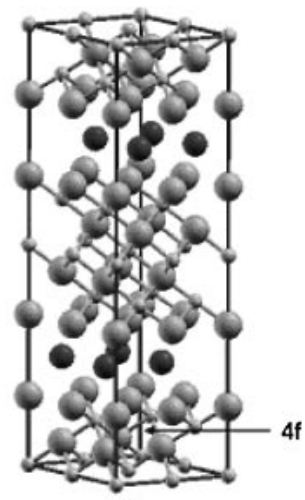

(b)
Fig. 1 The model of the carbon mono-vacancy at (a) $2 a$; and (b) $4 f$ site for $\mathrm{Ta}_{4} \mathrm{AlC}_{3}$.

and phase stability of $\mathrm{Ta}_{4} \mathrm{AlC}_{3}{ }^{11)}$ It is known that $\mathrm{Ta}_{4} \mathrm{AlC}_{3}$ crystallizes in the $\mathrm{PG}_{3} / \mathrm{mmc}$ space group and has 16 atoms in one unit cell. The thermodynamic ground state equilibrium structure of $\mathrm{Ta}_{4} \mathrm{AlC}_{3}$ has been determined to be the $\mathrm{Ti}_{4} \mathrm{AlN}_{3}$ like crystal structure, which is denoted as $\alpha$ polymorph. ${ }^{11,15)}$ In the present study, all the calculations are based on the $\alpha$ polymorph of $\mathrm{Ta}_{4} \mathrm{AlC}_{3}$. There are two different sites for carbon mono-vacancy in $\mathrm{Ta}_{4} \mathrm{AlC}_{3}$, namely $2 a$ and $4 f$ in the Wyckoff notation. As shown in Fig. 1, the carbon monovacancy structure was represented by a $2 \times 2 \times 1$ supercell containing 63 atoms after removing one $\mathrm{C}$ atom, corresponding to a sub-stoichiometry of $\mathrm{Ta}_{4} \mathrm{AlC}_{2.875}$. In our calculations, all the structural parameters for $\mathrm{Ta}_{4} \mathrm{AlC}_{3}$ with and without carbon vacancies were fully relaxed. As a first step, the lattice parameters for vacancy-free $\mathrm{Ta}_{4} \mathrm{AlC}_{3}$ were optimized. This was performed by applying hydrostatic pressure by changing the volumes while keeping the $c / a$ ratio constant. And then, with the optimized volume, the $c / a$ ratio was varied to find the values corresponding to the crystal energy minimum. Thirdly, the equilibrium positions of the internal Ta and $\mathrm{C}$ atoms were evaluated by calculating the Hellman-Feynman forces, ${ }^{16)}$ and the final forces on each atoms were less than $1 \mathrm{mRy} /$ a.u. Finally, for $\mathrm{Ta}_{4} \mathrm{AlC}_{3}$ with carbon mono-vacancy, 
Table 1 The calculated formation energy $\left(E_{\text {form }}\right)$ of carbon vacancy, total energy $\left(E_{\text {tot }}\right)$, volumes $(V)$, bulk modulus $(B)$ and density of states at the Fermi level $\left(N\left(E_{\mathrm{F}}\right)\right)$ for $\mathrm{Ta}_{4} \mathrm{AlC}_{3}$ with and without carbon mono-vacancy.

\begin{tabular}{lcccc}
\hline & $\begin{array}{c}E_{\text {form }} \\
(\mathrm{eV})\end{array}$ & $\begin{array}{c}E_{\text {tot }} \\
(\mathrm{Ry} / \text { unit cell })\end{array}$ & $\begin{array}{c}V \\
\left(\AA^{3} / \text { unit cell }\right)\end{array}$ & $\begin{array}{c}B \\
\left(E_{\mathrm{F}}\right) \\
(\mathrm{GPa})\end{array}$ \\
\hline $\mathrm{Ta}_{4} \mathrm{AlC}_{3}$ & $\cdots$ & -251446.621116 & 206 & 266 \\
$\mathrm{Ta}_{4} \mathrm{AlC}_{2.875}(2 a)$ & 2.22 & -251446.604596 & 205.6 & 261 \\
$\mathrm{Ta}_{4} \mathrm{AlC}_{2.875}(4 f)$ & 2.40 & -251446.591381 & 205.3 & 6.71 \\
\hline
\end{tabular}

the atomic arrangements around the carbon vacancy were also optimized by using calculated Hellmann-Feynman forces exerted on atoms in the vicinity of carbon vacancy.

\section{Results and Discussion}

As mentioned above, there are two different sites for carbon mono-vacancy in $\mathrm{Ta}_{4} \mathrm{AlC}_{3}$. To determine what defects are likely to form, the formation energy $E_{\mathrm{f}}$ for a carbon vacancy was determined by: ${ }^{13)}$

$$
E_{\mathrm{f}}=E_{\mathrm{t}}(\mathrm{v})+\mu_{\mathrm{C}}-E_{\mathrm{t}}
$$

where $E_{\mathrm{t}}(\mathrm{v})$ is the total energy of $\mathrm{Ta}_{4} \mathrm{AlC}_{3}$ with a carbon vacancy at $2 a$ and $4 f$ site respectively. $\mu_{\mathrm{C}}$ and $E_{\mathrm{t}}$ designate the total energy of an isolated $\mathrm{C}$ atom, and the total energy of vacancy-free $\mathrm{Ta}_{4} \mathrm{AlC}_{3}$, respectively. As listed in Table 1, the vacancy formation energy is calculated as $2.22 \mathrm{eV}$ for a carbon mono-vacancy at $2 a$ site, lower than $2.40 \mathrm{eV}$ for a carbon mono-vacancy at $4 f$ site. It is obvious that $\mathrm{C}$ vacancies at $2 a$ sites are energetically more favorable than at $4 f$ sites.

To determine the relative phase stabilities of $\mathrm{Ta}_{4} \mathrm{AlC}_{3}$ with and without carbon mono-vacancy, their total energies were also calculated and compared. The total energy of $\mathrm{Ta}_{4} \mathrm{AlC}_{3}$ without carbon vacancies is compared with the total energy of $\mathrm{Ta}_{4} \mathrm{AlC}_{3}$ with carbon vacancies plus the total energy of the extra carbon in the graphite structure, the most stable form of carbon, i.e.,

$$
E_{\mathrm{tot}}=E_{\mathrm{t}}(\mathrm{v})+x E\left(\mathrm{C}_{\text {graphite }}\right)
$$

The calculated ground state energies for $\mathrm{Ta}_{4} \mathrm{AlC}_{3}$ with and without carbon mono-vacancy are also listed in Table 1. It is seen that the total energy of $\mathrm{Ta}_{4} \mathrm{AlC}_{3}$ with carbon vacancies at $2 a$ sites is lower than at $4 f$ sites, which means that the former is energetically more stable. This conclusion is consistent with the analysis of the vacancy formation energy. However, the total energies of $\mathrm{Ta}_{4} \mathrm{AlC}_{3}$ with carbon vacancies are higher than the vacancy-free $\mathrm{Ta}_{4} \mathrm{AlC}_{3}$, which means that carbon vacancies are slightly difficult to form in this compound. Thus, high quality samples of $\mathrm{Ta}_{4} \mathrm{AlC}_{3}$ with stoichiometric composition will be easier to be prepared experimentally in comparison with other MAX phases. This result is supported by a recent experimental work on the crystal structure of $\mathrm{Ta}_{4} \mathrm{AlC}_{3}$, where the occupancy factors were refined and no significant deviations from the stoichiometric composition were found. ${ }^{10)}$

The equilibrium volume and the bulk modulus of $\mathrm{Ta}_{4} \mathrm{AlC}_{3}$ with and without carbon vacancy were calculated by fitting the Murnaghan equation of states to the energy-volume data. All calculated equilibrium volumes for $\mathrm{Ta}_{4} \mathrm{AlC}_{3}$ with and without carbon vacancies are within $1.8 \%$ from the experimental value. As listed in Table 1, introducing carbon

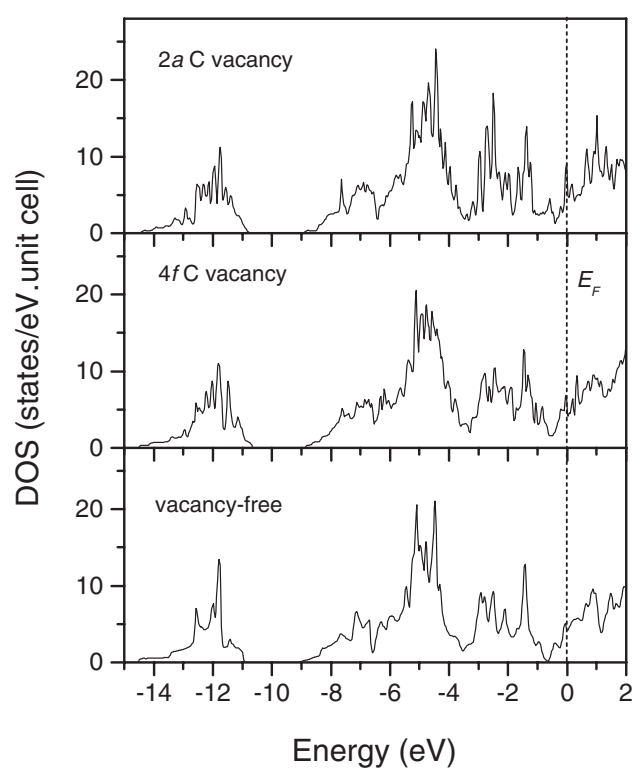

Fig. 2 The total density of states for $\mathrm{Ta}_{4} \mathrm{AlC}_{3}$ with and without carbon mono-vacancy.

mono-vacancy leads to a slight decrease in volume. The bulk modulus calculated for vacancy-free $\mathrm{Ta}_{4} \mathrm{AlC}_{3}$ is $266 \mathrm{GPa}$, in good agreement with the experimental value of $261 \mathrm{GPa}^{6}$ ) While the bulk modulus decreases to 261 , and $243 \mathrm{GPa}$ for $\mathrm{Ta}_{4} \mathrm{AlC}_{3}$ with $2 a$ and $4 f$ carbon vacancies respectively. It is obvious that the introduction of carbon vacancies at $4 f$ sites causes a larger decrease in bulk modulus.

The density of states (DOS) was calculated to see how the electronic states change in the presence of carbon vacancies. Figure 2 shows the DOS of $\mathrm{Ta}_{4} \mathrm{AlC}_{3}$ with carbon monovacancies at $2 a$ and $4 f$ sites respectively. For comparison, the DOS without carbon vacancies is also plotted. It can be seen that the total DOS of $\mathrm{Ta}_{4} \mathrm{AlC}_{3}$ with and without carbon vacancies exhibit some similar features. The states from about $-14 \mathrm{eV}$ to $-11 \mathrm{eV}$ are derived from the $\mathrm{C} 2 s$ and Ta $5 d$ states. The states just below the Fermi level are dominated by strong covalent bonding states of the Ta $5 d$ orbital and the $p$-derived orbitals of $\mathrm{Al}$ and $\mathrm{C}$. The states in the vicinity of the Fermi level are mainly occupied by Ta $5 d$ states, which contribute to the electronic conductivity. The detailed description of DOS can be found in our previous work. ${ }^{11)}$ Figure 3 shows the partial DOS of two nonequivalent Ta atoms nearest to the carbon vacancy. For comparison, the partial DOS of two nonequivalent Ta atoms in $\mathrm{Ta}_{4} \mathrm{AlC}_{3}$ without carbon vacancies is also shown in the figure. As can be seen, when carbon vacancies are included, some new peaks are seen to emerge just below the Fermi level. These vacancy peaks are of mainly Ta $5 d$ character. These peaks 


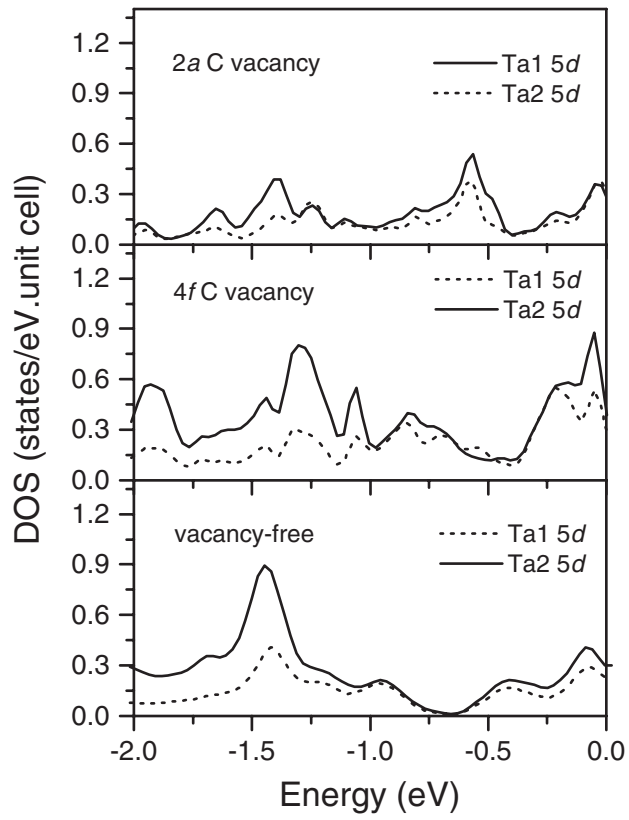

Fig. 3 The partial density of states showing the Ta-Ta $5 d$ hybridizations in $\mathrm{Ta}_{4} \mathrm{AlC}_{3}$ with and without carbon mono-vacancy.

are the so-called vacancy peaks that are associated with Ta-Ta bonds through the vacancy sites. Similar vacancy states were also found in the typical MAX phase, $\mathrm{Ti}_{3} \mathrm{SiC}_{2}$ previously. ${ }^{17)}$ Generally, the electronic conductivity has close correlation to the DOS at the Fermi level $\left(N\left(E_{\mathrm{F}}\right)\right)$. As can be seen from Table 1 , the value of $N\left(E_{\mathrm{F}}\right)$ increases from 4.18 states/eV.unit cell for $\mathrm{Ta}_{4} \mathrm{AlC}_{3}$ without carbon vacancies to 6.71, and 4.77 states/eV.unit cell for $\mathrm{Ta}_{4} \mathrm{AlC}_{3}$ with $2 a$ and $4 f$ carbon vacancies respectively. This result indicates that introducing carbon vacancies leads to the metallization of $\mathrm{Ta}_{4} \mathrm{AlC}_{3}$ and can improve the electronic conductivity of $\mathrm{Ta}_{4} \mathrm{AlC}_{3}$.

\section{Conclusions}

We have performed first-principles calculations to study the carbon mono-vacancy in $\mathrm{Ta}_{4} \mathrm{AlC}_{3}$. The $2 a$ sites are identified as the probable carbon vacancy sites in $\mathrm{Ta}_{4} \mathrm{AlC}_{3}$ based on the energetics of vacancy formation. It was found that introducing carbon vacancies decreased the phase stability of $\mathrm{Ta}_{4} \mathrm{AlC}_{3}$. There was no significant change in volume between $\mathrm{Ta}_{4} \mathrm{AlC}_{3}$ with and without carbon vacancy. The introduction of carbon vacancies decreased the bulk modulus of $\mathrm{Ta}_{4} \mathrm{AlC}_{3}$. It was observed that some vacancy states exist near the Fermi level. These vacancy states are associated with Ta-Ta bonds through the vacancy sites. Additionally, introducing carbon vacancies can improve the electronic conductivity of $\mathrm{Ta}_{4} \mathrm{AlC}_{3}$.

\section{REFERENCES}

1) M. W. Barsoum: Prog. Solid State Chem. 28 (2000) 201-281.

2) Z. M. Sun, Z. F. Zhang, H. Hashimoto and T. Abe: Mater. Trans. 43 (2002) 428-431.

3) Z. M. Sun, R. Ahuja and J. M. Schneider: Phys. Rev. B 68 (2003) 224112.

4) M. W. Barsoum, L. Farber, I. Levin, A. Procopio, T. El-Raghy and A. Berner: J. Am. Ceram. Soc. 82 (1999) 2545-2547.

5) J. P. Palmquist, T. El-Raghy, J. Höwing, O. Wilhelmsson and M. Sundberg: Abstract \#ICACC-S1-184-2006. In: 30th international conference on advanced ceramics and composites; 2006.

6) B. Manoun, S. K. Saxena, T. El-Raghy and M. W. Barsoum: Appl. Phys. Lett. 88 (2006) 201902.

7) Z. J. Lin, M. J. Zhuo, Y. C. Zhou, M. S. Li and J. Y. Wang: J. Mater. Res. 21 (2006) 2587-2592.

8) P. Eklund, J. P. Palmquist, J. Höwing, D. H. Trinh, T. El-Raghy, H. Högberg and L. Hultman: Acta Mater. 55 (2007) 4723-4729.

9) Z. J. Lin, M. J. Zhuo, Y. C. Zhou, M. S. Li and J. Y. Wang: J. Am. Ceram. Soc. 89 (2006) 3765-3769.

10) J. Etzkorn, M. Ade and H. Hillebrecht: Inorg. Chem. 46 (2007) 1410-1418.

11) Y. L. Du, Z. M. Sun, H. Hashimoto and W. B. Tian: Solid State Commun. 145 (2008) 461-464.

12) C. J. Rawn, M. W. Barsoum, T. El-Raghy, A. Procopio, C. M. Hoffmann and C. R. Hubbard: Mater. Res. Bull. 35 (2000) 17851796.

13) D. Music, R. Ahuja and J. M. Schneider: Appl. Phys. Lett. 86 (2005) 031911.

14) P. Blaha, K. Schwarz, G. K. H. Madsen, D. Kvasnicka and J. Luitz: WIEN2k, An Augmented Plane Wave plus Local Orbitals Program for Calculating Crystal Properties, (Techn. UniveresitatWien, Austria, 2007) ISBN 3-9501031-1-2.

15) J. Y. Wang, J. M. Wang, Y. C. Zhou, Z. J. Lin and C. F. Hu: Scripta Mater. 58 (2008) 1043-1046.

16) K. B. Panda and K. S. R. Chandran: Computational Mater. Sci. 35 (2006) 134-150.

17) N. I. Medvedeva, D. L. Novikov, A. L. Ivanovsky, M. V. Kuznetsov and A. J. Freeman: Phys. Rev. B 58 (1998) 16042-16050. 